\title{
Another look at number signals and preview sentences
}

\author{
FELICIA A. DIXON and JOHN A. GLOVER \\ Ball State University, Muncie, Indiana
}

\begin{abstract}
The combined effect of number signals and preview sentences on readers' recall of text material was examined. Subjects who read a passage in which both types of signals were used recalled significantly more signal-relevant information than did subjects in a number-signals only, a preview-sentence only, or a control condition. The effectiveness of number signals is discussed in terms of both encoding and retrieval effects. The influence of preview sentences is discussed in terms of how these devices may guide readers' attention.
\end{abstract}

Text signals are devices designed to indicate specific elements of text that are particularly important. Recently, there have been several studies of the effects of text signals (see Lorch, in press, for a review). In general, the literature indicates that signals do not change the overall amount of information readers recall, but they do increase the amount of signaled information remembered at the expense of unsignaled information (Britton, Glynn, Meyer, \& Penland, 1982; Irwin, 1982). This conclusion seems to hold for a variety of signaling devices, including number signals (Lorch \& Chen, 1986), headings (Lorch, in press), underlined and highlighted text (Glynn \& DiVesta, 1979), preview sentences (Lorch, 1985), and recall sentences (Glover et al., 1988). Despite the number of studies in the area, however, there have been no examinations of the combined effects of different types of signaling devices. The focus of the current study was on an examination of the combined effect of preview sentences and number signals.

Preview sentences signal information that readers will encounter at a later point in the material (e.g., "Below we will describe number signals"). Number signals are merely devices used to enumerate a set of to-be-learned points in a text (e.g., 1, 2, 3). Both types of signals are used extensively by writers and both have been found to influence patterns of readers' recall. The two types of signals, however, have not been contrasted, nor has anyone determined if their use in combination increases their influence on signal-relevant recall. We predicted that number signals would result in significantly greater levels of signal-relevant recall among subjects than would preview sentences. We made this prediction because number signals are physically present at the time the signal-relevant information is encoded, whereas representations of preview sentences must be held in a reader's memory. Furthermore, we expected this difference because number signals offer a very specific retrieval cue (e.g., "There

John Glover died in an accident in December 1989. Correspondence concerning this article may be addressed to Felicia A. Dixon, Burris Laboratory School, Ball State University, Muncie, IN 47306. are three things to remember, 1, 2, 3'). We also predicted, however, that a combined condition in which subjects encountered both preview sentences and number signals would result in the greatest level of signalrelevant recall.

\section{METHOD}

\section{Subjects and Setting}

The subjects were 62 junior and senior high school students drawn from a university laboratory school and a rural consolidated high school. All data were gathered in the students' regular classrooms.

\section{Materials}

A 24-paragraph essay originally developed by Bruning (1968) was employed. This essay describes a fictional African nation. Each paragraph is approximately $\mathbf{5 0}$ words in length and contains four sentences: a major idea sentence and three equally subordinate supporting sentences. Each sentence was constructed so that it contained one idea unit. The paragraphs were originally computer-generated and constructed so they could be placed in any order without influencing comprehension or recall (Bruning, 1968). Extensive research with these materials (e.g., Glover et al., 1988; Halpain, Glover, \& Harvey, 1985) has indicated that the 24 paragraphs are of equal memorability.

The paragraphs in the essay were ordered in four different ways. In each ordering, the last three paragraphs had number signals added to them to enumerate the three subordinate ideas in the paragraph. An example follows:

The major hope for industrial development in Mala probably is the potential water power. (1) There are just under 4,000 miles of flowing streams in Mala.

(2) The heads of all major rivers in Mala are over 3,000 feet above sea level.

(3) Six rivers have flows surpassing 50,000 cubic feet per second.

The three paragraphs congruent to those selected for enumeration in each different ordering had one of the subordinate sentences deleted and replaced by a preview sentence that signaled the upcoming content in the enumerated paragraphs. An example follows:

A special government 5-year plan is intended to increase hydroelectric output. The power output in Mala now is about 25 megawatts. At the end of 1995 the power output should be around 300 megawatts. More will be said about Mala's water power later in the chapter.

The paragraphs containing preview sentences appeared as the first three sentences in each ordering of the essay. Overall, then, each order of the essay contained three preview sentences (in the first three paragraphs) and three sets of number signals (in the last three paragraphs). After the basic essay was constructed, four forms appropriate to the four conditions were assembled. These forms contained no signals (control condition), preview sentences only (preview condition), number signals only (number-signal condition), and both preview sentences and number sig- 
nals (combined condition). Each of these four forms was typed in the three orders mentioned above.

\section{Procedure}

The subjects were randomly assigned to one of four conditions corresponding to the set of chapters they received: control, preview, number signals, or combined. Written directions informed the students that they were participating in a study in which their ability to remember reading material would be tested. They were then asked to read the essay carefully and prepare for a test over the content. The subjects were allowed to proceed at their own pace. The subjects were administered a free-recall posttest when they finished reading and studying the passage.

\section{RESULTS AND DISCUSSION}

The subjects' protocols were scored by identifying idea units in their protocols that matched those signaled in the enumerated paragraphs. In this process, each idea unit in these sentences was reduced to the fewest possible words from the text (or their synonyms) that would express the idea unit. The protocols were then scored for gist of meaning by two independent raters $(x=.98)$. Direct restatements of signaled passage contents as well as synonymous terms to indicate the same meaning were scored as correct recalls. Only recall of signaled content was scored. These data were then entered into a one-way analysis of variance employing conditions as the independent variable and signaled idea units recalled as the dependent variable. The results $\left[F(3,58)=77.08, M S_{\mathrm{e}}=\right.$ $1.97, p<.01]$ indicated a significant difference among the conditions. This outcome was then subjected to the Tukey HSD procedure $(\alpha=.05)$, which indicated that the subjects in the combined condition $(M=8.40, S D=$ 0.81 ) recalled significantly more of the signaled content than did the subjects in any of the other conditions. Furthermore, the subjects in the number-signal condition $(M=6.13, S D=2.09)$ recalled significantly more of the signaled content than did the subjects in either the preview-sentences condition $(M=3.88, S D=1.21)$ or the control condition $(M=0.93, S D=0.86)$. Finally, the subjects in the preview-sentences condition recalled significantly more of the signaled content than did the subjects in the control condition.

The results of the study confirmed our hypotheses. A combination of number signals and preview sentences was more influential in enhancing the recall of signal-relevant content than was either signal alone. Furthermore, number signals were more effective than preview sentences. Finally, preview sentences led to significantly greater levels of signal-relevant recall than did control conditions. Preview sentences seem to have their effect as a result of guiding readers' attention as they proceed through subsequent segments of text (Glover et al., 1988). This effect, however, depends on readers holding the gist of the preview sentences in active memory as they read. Number signals seem to have part of their effect by focusing attention to specific segments of text. Unlike preview sentences, however, number signals are physically present at the encoding of enumerated material and thus do not require readers to hold them in active memory. Number signals may also have their effect by indicating the importance of specific elements of text and by serving as a retrieval heuristic in free-recall situations. The combination of the two types of signals presumably results in some combination of the types of processing engendered by each signal alone.

\section{REFERENCES}

Britton, B. K., Glynn, S. M., Meyer, B. J. F., \& Penland, M. J. (1982). Effects of text structure on use of cognitive capacity during reading. Journal of Educational Psychology, 74, 51-61.

BRUNING, R. H. (1968). Effects of review and test-like events within the learning of prose materials. Journal of Educational Psychology, 59, 16-19.

Glover, J. A., Dinnel, D. L., Halpain, D. R., McKee, T. K., CorKILL, A. J., \& WISE, S. L. (1988). Effects of across-chapters signals on recall of text. Journal of Educational Psychology, 80, 3-15.

GlynN, S. M., \& DiVesta, F. J. (1979). Control of prose processing via instructional and typographical cues. Journal of Educational Psychology, 71, 595-603.

Halpain, D. R., Glover, J. A., \& Harvey, A. L. (1985). Differential effects of higher and lower order questions: Attention hypotheses. Journal of Educational Psychology, 77, 703-715.

IRWIN, J. W. (1982). The effects of coherence explicitness on college readers' prose comprehension. Journal of Reading Behavior, 14, 275-284.

LORCH, R. F., JR. (1985). Effects on recall of signals to text organization. Bulletin of the Psychonomic Society, 23, 374-376.

LORCH, R. F., JR. (in press). Text signaling devices and their effects on memory. Educational Psychology Review.

LORCH, R. F., JR., \& CHEN, A. H. (1986). Effect of number signals on reading and recall. Journal of Educational Psychology, 78, 263-270.

(Manuscript received December 8, 1989.) 